\title{
Inviscid two dimensional vortex dynamics and a soliton expansion of the sinh-Poisson equation
}

\author{
K. W. Chow, ${ }^{\text {a) }}$ N. W. M. Ko, and R. C. K. Leung \\ Department of Mechanical Engineering, University of Hong Kong, Pokfulam Road, Hong Kong \\ S. K. Tang \\ Department of Building Services Engineering, Hong Kong Polytechnic University, Hunghom, Hong Kong
}

(Received 2 September 1997; accepted 23 January 1998)

\begin{abstract}
The dynamics of inviscid, steady, two dimensional flows is examined for the case of a hyperbolic sine functional relation between the vorticity and the stream function. The 2-soliton solution of the sinh-Poisson equation with complex wavenumbers will reproduce the Mallier-Maslowe pattern, a row of counter-rotating vortices. A special 4-soliton solution is derived and the corresponding flow configuration is studied. By choosing special wavenumbers complex flows bounded by two rigid walls can result. A conjecture regarding the number of recirculation regions and the wavenumber of the soliton expansion is offered. The validity of the new solution is verified independently by direct differentiation with a computer algebra software. The circulation and the vorticity of these novel flow patterns are finite and are expressed in terms of well defined integrals. The questions of the linear stability and the nonlinear evolution of a finite amplitude disturbance of these steady vortices are left for future studies. (C) 1998 American Institute of Physics. [S1070-6631(98)01805-4]
\end{abstract}

\section{INTRODUCTION}

The dynamics of vorticity plays an important role in fluid mechanics by providing a convenient, efficient and instructive perspective of the flow. ${ }^{1,2}$ Recently there has been a strong interest in studying two dimensional (2D) vortex structures under various conditions. Such problems are not only of fundamental fluid dynamical interest but will also provide relevant information on turbulent flows. Among the various possible applications of such coherent vortices are geophysical flows, ${ }^{3}$ rotating and stratified fluids ${ }^{4}$ and fluid layers excited by electromagnetic forces. ${ }^{5}$

For inviscid, steady 2D flows without body force, one general solution of fluid motions is $(\omega, \psi$ being the vorticity and the stream function, respectively): $:^{1,2}$

$$
-\omega=\nabla^{2} \psi=\left(\frac{\partial^{2} \psi}{\partial x^{2}}+\frac{\partial^{2} \psi}{\partial y^{2}}\right)=f(\psi) .
$$

$f$ needs to be differentiable but otherwise arbitrary. Point vortices, vortex sheets and other singular distributions of vorticity have been studied extensively in the literature. The goal of the present work is to derive new, globally smooth, exact solutions of (1.1) for the special case $f(\psi)$ $=-\sinh \psi$. Solutions for $f$ being constant are discussed in standard references. ${ }^{1,2}$ A vortex patch is a connected region of finite area containing uniform vorticity surrounded by an irrotational fluid. The evolution of a vortex patch can be treated by the methods of contour dynamics and Schwarz functions. A simple example of a vortex patch is a Rankine vortex, a circular region of vortical fluid of a fixed radius in an otherwise unbounded irrotational fluid. Kirchhoff extended the reasoning, and gave an expression for an elliptical

${ }^{a)}$ Corresponding author. Electronic mail: kwchow@hkusua.hku.hk patch rotating with a steady angular velocity. Moore and Saffman further generalized the solution to a vortex patch in a uniform straining field. Kida included the effect of time dependence in the straining field. An extensive discussion of the dynamics of these elliptical vortices is given by Saffman. $^{2}$

The stability of these configurations is not a trivial matter and has also received extensive treatment. ${ }^{6,7}$ The properties and dynamics of a single, elliptical vortex patch have continued to attract attention recently. Indeed the Lagrangian trajectories around the vortex might become chaotic. ${ }^{8}$

Properties of two or an array of vortex patches are usually handled numerically. Critical questions include but are not limited to

(i) the shape of the patches as the size of each individual unit increases from being very small through a finite measure, and finally to the case of touching patches, (ii) the stability of such arrangements. ${ }^{2}$

The case of linear $f$ has also received tremendous attention lately. A Lamb dipole is the simplest example. ${ }^{2}$ An external irrotational flow with a suitably chosen free stream speed encloses a fluid endowed with vorticity. The circular boundary has a radius determined by the zero of a Bessel function. Chaplygin probably investigated a similar problem independently at about the same time as Lamb did, but he considered the case where the interior flow is not symmetrical as well. ${ }^{9}$ Analogous calculations have been performed for the quasi-geostrophic system of equations. The resulting solutions, the modons, constitute an active field of research. ${ }^{3}$

Nonlinear cases documented in the literature include

(i) $f(\psi)=\exp (-2 \psi)$ : the Liouville equation. ${ }^{10}$ The Stuart vortices constitute a cat-eye pattern, and represent a con- 
tinuous family of solutions from a shear layer to a rectilinear array of point vortices.

(ii) $f(\psi)=-\sinh \psi$ : known either as the sinh-Gordon or the sinh-Poisson (shP) equation. ${ }^{11,12}$

Mallier and Maslowe showed that a row of counter rotating vortices constitutes a special solution of shP. ${ }^{11}$ Pasmanter examined certain aspects of the controversies surrounding the precise relationship between the vorticity and the stream function in decaying two dimensional turbulence. ${ }^{12}$ He also gave special axisymmetric solutions of shP in an unbounded fluid in terms of known differential equations. The stability of the Mallier-Maslowe configuration was investigated by Dauxois et al. ${ }^{13}$

(iii) $f(\psi)=a \psi+b \psi^{3}$ : There is a widely held belief in the nonlinear science community that the two dimensional nonlinear Schrödinger equation,

$$
i A_{t}+A_{x x}+A_{y y} \pm A^{2} A^{\star}=0,
$$

is not integrable. 2-soliton solution does not exist. Consequently a time harmonic solution $A(x, y, t)=u(x, y)$ $\times \exp (-i \Omega t)$,

$$
u_{x x}+u_{y y}+\Omega u \pm u^{3}=0,
$$

should not possess any special property. Indeed the dipole solution must be computed numerically. ${ }^{14}$ Numerical integration of the Euler equations shows that the dipole is unstable. ${ }^{14}$ However, the relation between the integrability of $\psi_{x x}+\psi_{y y}=f(\psi)$ and the stability of the associated vortical patterns is unlikely to be simple.

Mathematically shP is closely related to the elliptic version of the sine-Gordon $(\mathrm{sG})$ equation

$$
\psi_{x x}+\psi_{y y}=\sin \psi
$$

Studies of shP and sG actually have a long history in the literature of nonlinear waves. An expression for an arbitrary number of solitons for $\mathrm{sG}$ is available. ${ }^{15}$ Periodic solutions of $\mathrm{sG}$ in terms of Riemann theta functions and the scattering transform can be found. ${ }^{16}$ Travelling waves for the $(2+1)(2$ spatial and 1 temporal) dimensional $\mathrm{sG}$ can be described in terms of classical elliptic functions. ${ }^{17}$ Some of these can reduce to a steady state case and hence might correspond to solutions in inviscid vortex dynamics. For shP a periodic solution in terms of theta functions and the inverse methods is obtained for a square with homogeneous boundary conditions. $^{18}$

Recently it has been shown that the Mallier-Maslowe vortices can be obtained as a 2-soliton solution of shP with complex conjugate wavenumbers. ${ }^{19}$ A special solution from a 3-soliton expansion of sG and some improved doubly periodic solutions were also given. The goal of the present work is to present a special 4-soliton expansion of shP and to study the consequence in vortex dynamics.

For completeness the calculations reported earlier in the literature ${ }^{19}$ are reproduced here. The sinh-Poisson equation

$$
\psi_{x x}+\psi_{y y}=-\sinh \psi
$$

has a 2-soliton solution

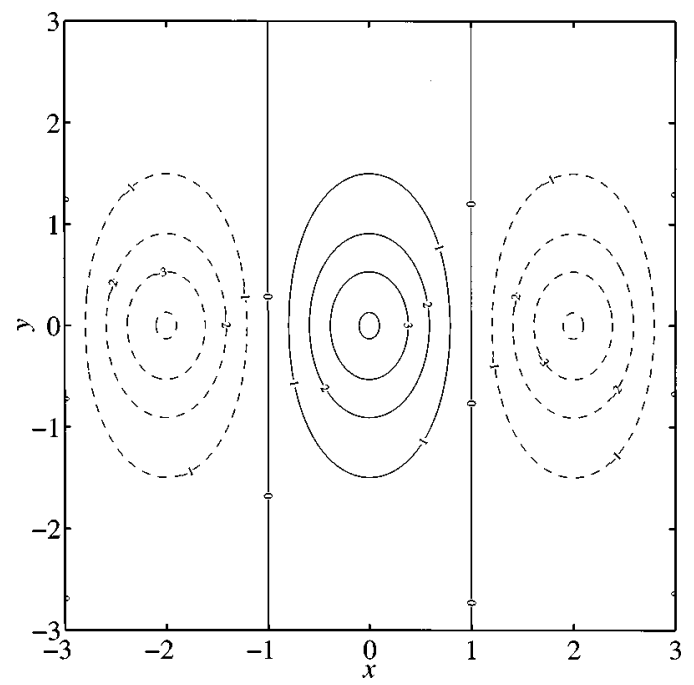

FIG. 1. Streamlines for the Mallier-Maslowe pattern (1.6), $\sqrt{1+k^{2}}=\pi / 2$.

$$
\begin{aligned}
& \psi=4 \tanh ^{-1}\left(\frac{g_{2}}{f_{2}}\right), \quad g_{2}=\exp \left(\phi_{1}\right)+\exp \left(\phi_{2}\right), \\
& f_{2}=1+m \exp \left(\phi_{1}+\phi_{2}\right), \\
& \phi_{n}=p_{n} x+q_{n} y, \quad p_{n}^{2}+q_{n}^{2}=-1, \quad n=1,2, \\
& m=-\frac{\left(p_{1}-p_{2}\right)^{2}+\left(q_{1}-q_{2}\right)^{2}}{\left(p_{1}+p_{2}\right)^{2}+\left(q_{1}+q_{2}\right)^{2}} .
\end{aligned}
$$

By choosing $p_{1}=p_{2}^{\star}=i \sqrt{1+k^{2}}, q_{1}=q_{2}=k, k$ real, one obtains a form equivalent to the Mallier-Maslowe vortices. In the present notations this expression is

$$
\begin{aligned}
& \psi_{x x}+\psi_{y y}=-\sinh \psi \\
& \psi=4 \tanh ^{-1}\left[\frac{k \cos \left(\sqrt{1+k^{2}} x\right)}{\sqrt{1+k^{2}} \cosh (k y)}\right] .
\end{aligned}
$$

For comparisons with the main results presented later Fig. 1 shows the flow pattern associated with (1.6).

Due to the restriction $p_{n}^{2}+q_{n}^{2}=-1$, either $p_{n}$ or $q_{n}$ must be complex. To ensure real solutions the wavenumbers $\left(p_{n}, q_{n}\right)$ must be taken in complex conjugate pairs. Hence only solitons of even orders are viable solutions of vortex dynamics. In contrast, the dispersion relation for the sineGordon equation is $p_{n}^{2}+q_{n}^{2}=+1$, and thus multi-soliton of all orders are possible candidates. However, in view of the importance and relevance of shP in earlier experimental and computational works in fluid dynamics, we shall focus on shP in this paper. Although one can argue that solutions of $\mathrm{sG}$ and shP are related by the mapping

$$
u_{x x}+u_{y y}=\sin u, \quad v_{x x}+v_{y y}=\sinh v, \quad v=i u .
$$

The purely imaginary nature of this transformation does not allow any known solution of $\mathrm{sG}$ to translate directly into flow patterns. A Bäcklund transformation exists between real solutions of sG and shP. ${ }^{12}$ However, it is a highly nontrivial task to generate solutions of shP from the known multi- 
soliton solutions of $\mathrm{sG}$ through this transformation. Consequently, one might have to start from first principles in deriving solutions for shP.

Besides vortex dynamics a very important application of shP is the theory of 2D turbulence. The dynamics of two dimensional line vortices can be treated by the methods of statistical mechanics. The sinh Poisson equation is satisfied by the most probable state of a system of point vortices in thermal equilibrium. Hence (1.3) will provide relevant information on the relaxed state of $2 \mathrm{D}$, high Reynolds number turbulence. $^{20}$

The picture now is that the lowest permissible member of the shP hierarchy, the 2-soliton, reproduces the MallierMaslowe pattern. We shall derive a special 4-soliton expansion in Sec. II and study the associated flow patterns in Sec. III.

\section{A SPECIAL 4-SOLITON EXPANSION}

The search for multi-soliton of nonlinear evolution equations is by now a well established discipline. ${ }^{15}$ For the present purpose we shall employ the Hirota bilinear operator

$$
\begin{aligned}
D_{x}^{m} D_{t}^{n} g . f= & \left(\frac{\partial}{\partial x}-\frac{\partial}{\partial x^{\prime}}\right)^{m}\left(\frac{\partial}{\partial t}-\frac{\partial}{\partial t^{\prime}}\right)^{n} \\
& \times\left. g(x, t) f\left(x^{\prime}, t^{\prime}\right)\right|_{x=x^{\prime}, t=t^{\prime}} .
\end{aligned}
$$

shP (1.3) can now be rewritten in the bilinear format:

$$
\begin{aligned}
& \psi=4 \tanh ^{-1}\left(\frac{g}{f}\right)=2 \log \left(\frac{f+g}{f-g}\right), \\
& \left(D_{x}^{2}+D_{y}^{2}\right)(g \cdot g+f . f)=0, \quad\left(D_{x}^{2}+D_{y}^{2}\right) g . f=-g f .
\end{aligned}
$$

The identity

$$
2(\log F)_{x x}=\frac{D_{x}^{2} F \cdot F}{F^{2}}
$$

has been used. $D$ is a linear operator. Appropriate functional forms for the expansion of this type of bilinear equations are known. ${ }^{15}$ The key to the search is to ensure that the expansion actually truncates at one particular level, e.g., (1.4), (1.5) satisfy (2.3) through the identity

$$
\begin{aligned}
& D_{x}^{m} D_{y}^{n} \exp (a x+r y) \cdot \exp (b x+s y) \\
& \quad=(a-b)^{m}(r-s)^{n} \exp [(a+b) x+(r+s) y] .
\end{aligned}
$$

Tedious and oppressive algebra is usually involved in a higher order search, but that difficulty is largely mitigated currently by the widespread use of commercial computer algebra software, e.g., MATHEMATICA. Since the dispersion re- lation (1.5) dictates that complex conjugate wavenumbers must eventually be used, one searches only for this special style of 4-soliton expansion. The appropriate expansion for shP (2.1)-(2.3) that will form the central theme of the present work is

$$
\begin{aligned}
f= & 1+m_{12} \exp \left(\phi_{1}+\phi_{2}\right)+m_{13} \exp \left(\phi_{1}+\phi_{3}\right) \\
& +m_{14} \exp \left(\phi_{1}+\phi_{4}\right)+m_{23} \exp \left(\phi_{2}+\phi_{3}\right) \\
& +m_{24} \exp \left(\phi_{2}+\phi_{4}\right)+m_{34} \exp \left(\phi_{3}+\phi_{4}\right) \\
& +m_{12} m_{13} m_{14} m_{23} m_{24} m_{34} \exp \left(\phi_{1}+\phi_{2}+\phi_{3}+\phi_{4}\right) \\
g= & \exp \left(\phi_{1}\right)+\exp \left(\phi_{2}\right)+\exp \left(\phi_{3}\right)+\exp \left(\phi_{4}\right) \\
& +\chi_{1} \exp \left(\phi_{2}+\phi_{3}+\phi_{4}\right)+\chi_{2} \exp \left(\phi_{1}+\phi_{3}+\phi_{4}\right) \\
& +\chi_{3} \exp \left(\phi_{1}+\phi_{2}+\phi_{4}\right)+\chi_{4} \exp \left(\phi_{1}+\phi_{2}+\phi_{3}\right)
\end{aligned}
$$

Phase factors must occur in complex conjugate pairs

$$
\begin{array}{ll}
\phi_{1}=\phi=p_{1} x+q_{1} y, & \phi_{3}=\phi^{\star}, \\
\phi_{2}=\psi=p_{2} x+q_{2} y, & \phi_{4}=\psi^{\star} .
\end{array}
$$

By repeated use of the identity (2.5) our contribution is to prove that the expansion (2.6), (2.7) will actually truncate at this level at least for the special case of $q_{1}=\alpha$ (real), $q_{2}$ $=\beta$ (real). Through these intermediate calculations the remaining parameters can now be determined:

$$
\begin{aligned}
& p_{n}^{2}+q_{n}^{2}=-1, \quad n=1,2, \quad p_{1}=i \sqrt{1+\alpha^{2}}, \quad q_{1}=\alpha, \\
& p_{2}=i \sqrt{1+\beta^{2}}, \quad q_{2}=\beta, \\
& m_{i j}=\frac{S_{i j}+1}{S_{i j}-1}, \quad S_{i j}=p_{i} p_{j}+q_{i} q_{j}, \\
& \chi_{1}=\chi_{3}=n_{1}=m_{12} m_{14} m_{24}, \quad \chi_{2}=\chi_{4}=n_{2}=m_{12} m_{13} m_{23} .
\end{aligned}
$$

Straightforward algebra now reveals a concrete final answer for the stream function and the vorticity:

$$
\begin{aligned}
g= & 2\left\{\exp (\alpha y) \cos \left(\sqrt{1+\alpha^{2}} x\right)+\exp (\beta y) \cos \left(\sqrt{1+\beta^{2}} x\right)\right. \\
& +\left(\frac{\alpha-\beta}{\alpha+\beta}\right)^{2} \exp ((\alpha+\beta) y) \\
& \times\left[\left(1+\frac{1}{\beta^{2}}\right) \exp (\beta y) \cos \left(\sqrt{1+\alpha^{2}} x\right)\right. \\
& \left.\left.+\left(1+\frac{1}{\alpha^{2}}\right) \exp (\alpha y) \cos \left(\sqrt{1+\beta^{2}} x\right)\right]\right\}
\end{aligned}
$$

$$
\begin{aligned}
f= & 1+\left(1+\frac{1}{\alpha^{2}}\right) \exp (2 \alpha y)+\left(1+\frac{1}{\beta^{2}}\right) \exp (2 \beta y)+2\left[\frac{\alpha \beta-\sqrt{1+\alpha^{2}} \sqrt{1+\beta^{2}}+1}{\alpha \beta-\sqrt{1+\alpha^{2}} \sqrt{1+\beta^{2}}-1}\right] \exp ((\alpha+\beta) y) \cos \left(\left(\sqrt{1+\alpha^{2}}+\sqrt{1+\beta^{2}}\right) x\right) \\
& +2\left[\frac{\alpha \beta+\sqrt{1+\alpha^{2}} \sqrt{1+\beta^{2}}+1}{\alpha \beta+\sqrt{1+\alpha^{2}} \sqrt{1+\beta^{2}}-1}\right] \exp ((\alpha+\beta) y) \cos \left(\left(\sqrt{1+\alpha^{2}}-\sqrt{1+\beta^{2}}\right) x\right)+\left(1+\frac{1}{\alpha^{2}}\right)\left(1+\frac{1}{\beta^{2}}\right)\left(\frac{\alpha-\beta}{\alpha+\beta}\right)^{4} \exp (2 \alpha y+2 \beta y)
\end{aligned}
$$




$$
\begin{aligned}
& \psi(x, y, \alpha, \beta)=4 \tanh ^{-1}\left(\frac{g}{f}\right), \\
& \psi_{x x}+\psi_{y y}=-\omega=-\sinh \psi .
\end{aligned}
$$

(2.10), (2.11) constitute the main result of the present work. Note that $(2.10),(2.11)$ reduce to (1.6) for the special case of $\alpha=\beta$, provided that a simple translation

$$
y \rightarrow y^{\prime}+\frac{1}{\alpha} \log \left(\frac{\alpha}{2 \sqrt{1+\alpha^{2}}}\right)
$$

is made (and primes are dropped).

Furthermore (2.10), (2.11) possess a number of symmetries:

$$
\begin{aligned}
& \psi(x, y, \alpha, \beta)=\psi(x, y, \beta, \alpha), \\
& \psi(x, y, \alpha, \beta)=\psi(x,-y,-\alpha,-\beta), \\
& \psi(x, y, \alpha, \beta)=\psi(-x, y, \alpha, \beta) .
\end{aligned}
$$

(2.14) implies that it is sufficient to consider $\beta>\alpha$ in the subsequent discussion. Apart from illustrating the reflection properties of $(2.10),(2.11),(2.15),(2.16)$ can be used to classify the $(\alpha, \beta)$ parameter plane. More precisely, $(2.15)$ implies that one only needs to consider two cases: $\alpha>0, \beta$ $>0$ and $\alpha>0, \beta<0$. The variation of the flow patterns with respect to changes in $\beta$ is documented in Sec. III.

Confirmation. Exact solutions are generally rare in fluid mechanics, and (2.10), (2.11) are certainly not intuitively obvious solutions of the equations of motion. Hence it is worthwhile to confirm the validity of $(2.10)$, (2.11) by an independent procedure. We shall in fact prove that (2.12) is satisfied by direct differentiation. Such lengthy calculations are handled by the software MATHEMATICA. We shall verify that

$$
\begin{gathered}
{\left[4 \tanh ^{-1}\left(\frac{g}{f}\right)\right]_{x x}+\left[4 \tanh ^{-1}\left(\frac{g}{f}\right)\right]_{y y}=-\frac{4 f g\left(f^{2}+g^{2}\right)}{\left(f^{2}-g^{2}\right)^{2}}} \\
\left(=-\sinh \left[4 \tanh ^{-1}\left(\frac{g}{f}\right)\right]\right) \cdot
\end{gathered}
$$

We first perform the differentiation on the left symbolically using $g, g_{x}, g_{y}, g_{x x}, g_{y y}$ and the counterparts for $f$. The common denominator $\left(f^{2}-g^{2}\right)^{2}$ is then removed. The precise forms for the derivatives of $f$ and $g$ are now computed in terms of exponential functions in $y$ and trigonometric functions in $x$. The computations are again performed by MATHEMATICA utilizing (2.10), (2.11). These well defined expressions are then substituted into the numerators of both sides of (2.17).

Finally, we expand each side in terms of the powers $U^{m} V^{n}$, where $U=\exp (\alpha y), V=\exp (\beta y), m, n$ integers. How an arbitrary piece of commercial software will deal with complex trigonometric formulas in general is uncertain, and unwise applications of the compound angle formulas may actually increase the execution time of the program. Hence it might be advisable to reduce all intermediate calculations to a purely algebraic nature by using well known expressions, e.g.,

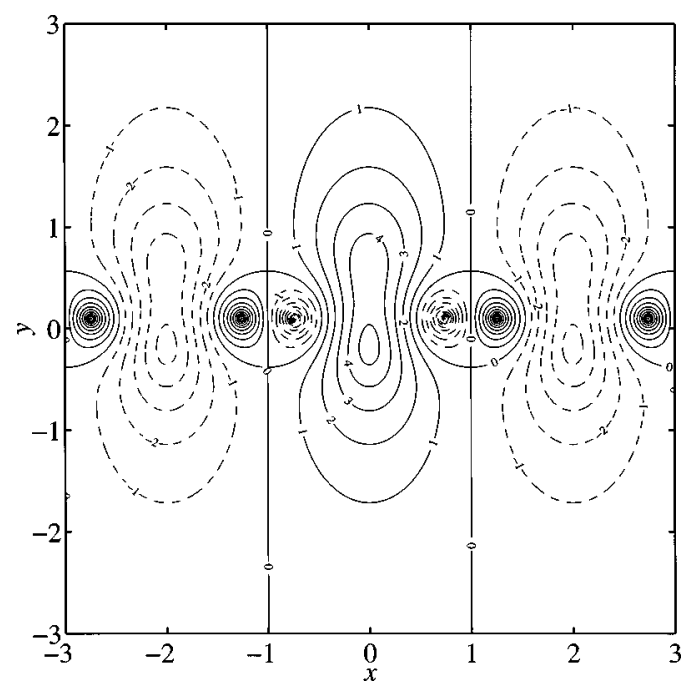

FIG. 2. Streamlines for (2.10), (2.11) for $\sqrt{1+\alpha^{2}}=\pi / 2, \sqrt{1+\beta^{2}}=3 \pi / 2$, $\beta>0$.

$$
\begin{aligned}
& \cos \left(\sqrt{1+\alpha^{2}} x\right)=\frac{1}{2}\left(P+\frac{1}{P}\right), \quad P=\exp \left(i \sqrt{1+\alpha^{2}} x\right) \\
& \sin \left(\sqrt{1+\beta^{2}} x\right)=\frac{1}{2 i}\left(Q-\frac{1}{Q}\right), \quad Q=\exp \left(i \sqrt{1+\beta^{2}} x\right), \\
& \sin \left[\left(\sqrt{1+\alpha^{2}}-\sqrt{1+\beta^{2}}\right) x\right]=\frac{1}{2 i}\left(\frac{P}{Q}-\frac{Q}{P}\right) .
\end{aligned}
$$

There are about twenty terms of the form $U^{m} V^{n}$ with coefficients as lengthy, but well defined, algebraic functions of $P$ and $Q$. We verify that identical powers from both sides match exactly. About ten calculations require somewhere from 10 to 20 min of computer time. The validity of (2.10), (2.11) is thus confirmed.

\section{RESULTS}

To perform a comprehensive search on the properties of (2.10)-(2.12) in the $(\alpha, \beta)$ plane will be a difficult exercise. An additional constraint is that real, physical solutions are obtained only for $|g|<|f|$ due to the inverse hyperbolic tangent. We focus on several extensions of known solutions.

\section{A. Vortices in a channel}

(1) $\sqrt{1+\alpha^{2}}=\pi / 2 \quad(\alpha=1.21136), \sqrt{1+\beta^{2}}=3 \pi / 2 \quad(\beta$ $=4.60506$ ). The vertical lines $x=N, N=$ an odd integer, are streamlines, as there is no horizontal (normal) component of velocity along such lines. Hence they can be replaced by rigid walls if necessary. Only pictures for the streamlines will be shown, as diagrams for the vorticity contours are basically identical. Note that fluids carrying vorticity of different signs protrude into the original Mallier-Maslowe pattern (Fig. 2).

This might be one form of the phenomenon of 'filamentation' studied earlier, ${ }^{2}$ but now the intruding fluids assume 


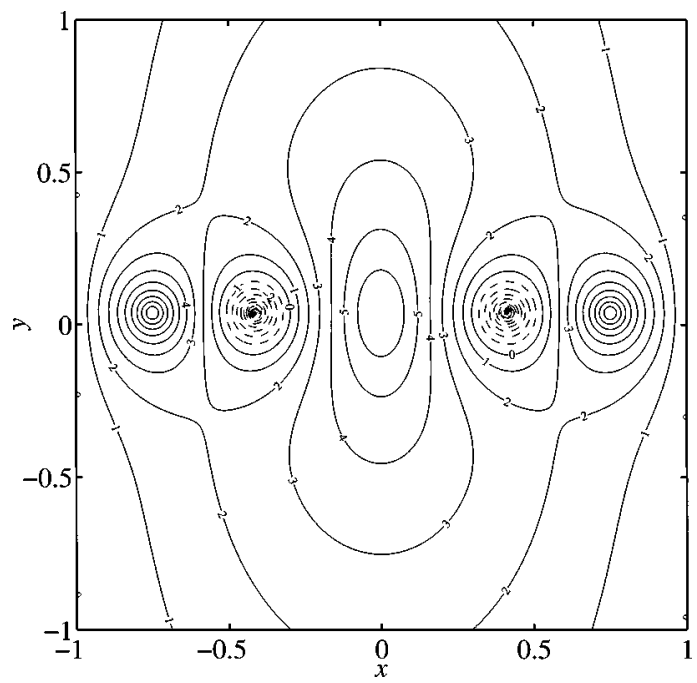

FIG. 3. Streamlines for $(2.10),(2.11)$ for $\sqrt{1+\alpha^{2}}=\pi / 2, \sqrt{1+\beta^{2}}=5 \pi / 2$, $\beta>0$.

an oval shape rather than an elongated finger. Furthermore, fluids on both sides of the boundary are endowed with vorticity.

The relationship between the number of solitons and the external appearance of the flow is subtle, and pictures from the low wavenumbers regime can be deceptive. In the Mallier-Maslowe solution there are two types of closed streamlines (one with $\psi>0$, one with $\psi<0$ ) before the pattern repeats itself (Fig. 1). In the present case there are clearly four kinds of recirculation regions in Fig. 2 (by the shape and sign of the streamlines). One is tempted to link the number of families of closed streamlines with the order of the soliton expansion. That such a correlation might not exist (or at least, not a simple one) is illustrated next.

(2) $\sqrt{1+\alpha^{2}}=\pi / 2 \quad(\alpha=1.21136), \sqrt{1+\beta^{2}}=5 \pi / 2 \quad(\beta$ $=7.79006)$. The lines $x=N(N=$ odd integer $)$ are again streamlines. However, there are now five recirculating regions inside a lemon shaped streamline (Fig. 3).

(3) $\sqrt{1+\alpha^{2}}=M \pi / 2, \sqrt{1+\beta^{2}}=N \pi / 2, M, N$ odd integers. As pointed out earlier it is now sufficient to consider $M<N$. For comparison we present

(i) $\quad M=1, N=7$ (Fig. 4),

(ii) $\quad M=1, N=9$ (Fig. 5),

(iii) $M=3, N=5$ (Fig. 6),

(iv) $\quad M=3, N=7$ (Fig. 7),

(v) $M=5, N=7$ (Fig. 8),

(vi) $M=5, N=9$ (Fig. 9).

By examining these figures we suggest a rule which might govern the dynamics.

A mathematical conjecture. The lines $x= \pm 1$ remain streamlines for the choice $\sqrt{1+\alpha^{2}}=M \pi / 2, \sqrt{1+\beta^{2}}$ $=N \pi / 2, M, N$ odd integers. Obviously the flow patterns will get increasingly complex as $M$ and $N$ become larger. (2.14) implies that it is sufficient to consider $M<N$. For the purpose of the present discussion we define a fundamental recirculation region (FRR) in the following manner. A FRR consists of a sequence of closed streamlines with strictly

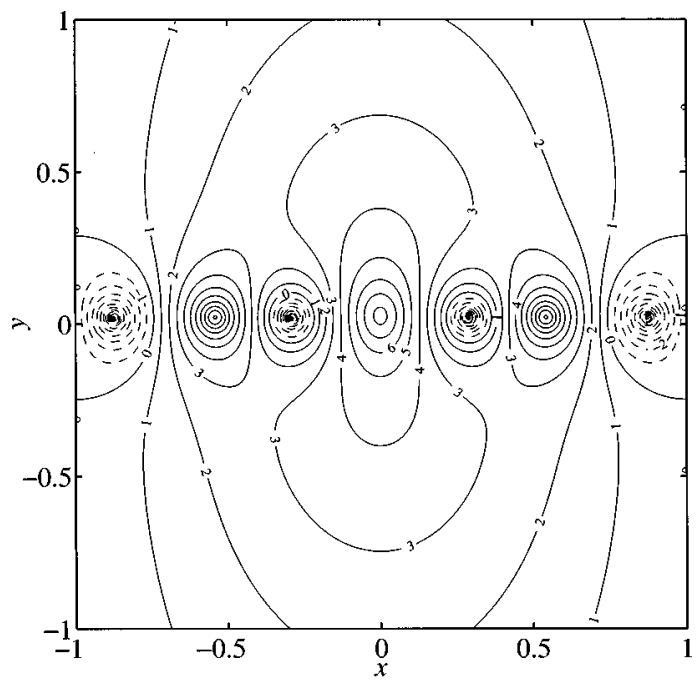

FIG. 4. Streamlines for (2.10), (2.11) for $\sqrt{1+\alpha^{2}}=\pi / 2, \sqrt{1+\beta^{2}}=7 \pi / 2$, $\beta>0$.

monotonic increasing (or decreasing) $\psi$ as the area enclosed by the streamlines shrinks to zero.

Consider Fig. 3 as an example. The eddy in the center qualifies as a fundamental recirculation region, since $\psi$ increases strictly from about 3 as each streamline decreases in size. The family of streamlines enclosing all five eddies, e.g., the $\psi=1$ to $\psi=2$ sequence close to the walls $x= \pm 1$, must be rejected. The reason is that both regions of increasing and decreasing values of $\psi$ are enclosed, and hence the variation of $\psi$ is not monotonic.

We now offer the following conjecture which consists of several parts:

The special 4-soliton solution of the sinh Poisson equation, (2.10), (2.11), will contain for $\sqrt{1+\alpha^{2}}$ $=M \pi / 2$ and $\sqrt{1+\beta^{2}}=N \pi / 2, N>M>1$ being positive odd integers, $M+N$ fundamental recirculation regions between the streamlines $x= \pm 1$.

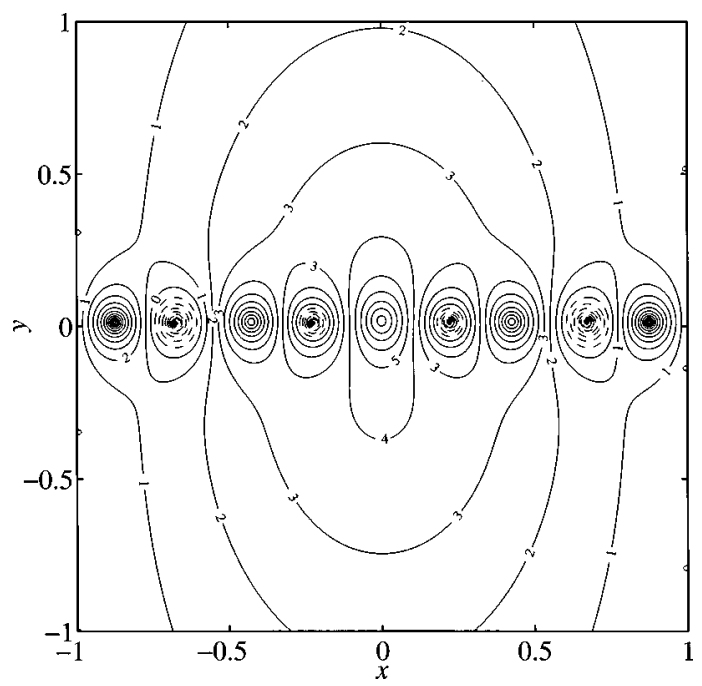

FIG. 5. Streamlines for (2.10), (2.11) for $\sqrt{1+\alpha^{2}}=\pi / 2, \sqrt{1+\beta^{2}}=9 \pi / 2$, $\beta>0$. 


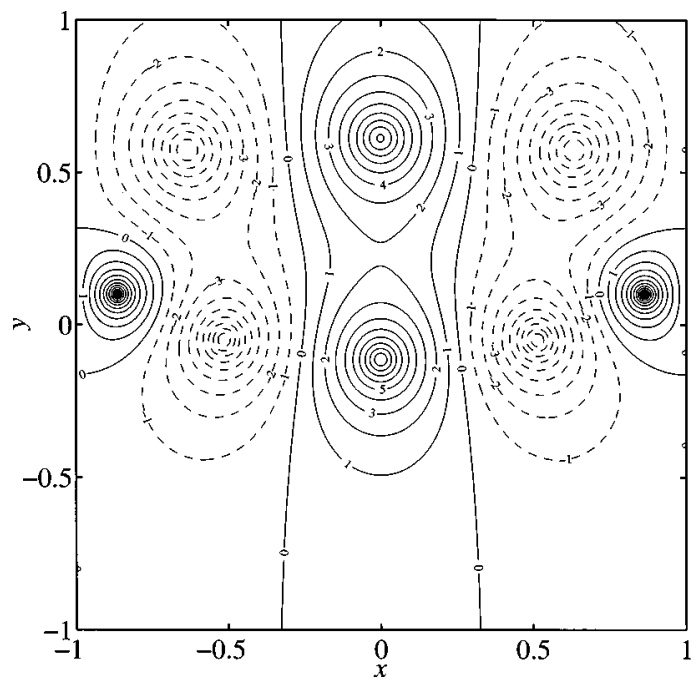

FIG. 6. Streamlines for (2.10), (2.11) for $\sqrt{1+\alpha^{2}}=3 \pi / 2, \sqrt{1+\beta^{2}}=5 \pi / 2$, $\beta>0$.

(ii) If $M=1$ and $N>1$, the number of fundamental recirculation regions is reduced to $N$ for $\alpha>0, \beta>0$, or one unit less than the case for $M>1$.

(iii) If $M=1$ and $N>1$, the number of fundamental recirculation regions remains $N+1$ for $\alpha>0, \beta<0$, due to the appearance of a new unit below the main sequence of vortices (Figs. 10, 11).

(iv) The Mallier-Maslowe pattern corresponds to the degenerate case $M=1, N=1, \alpha=\beta>0$, and there is then exactly one family of closed streamlines between $x= \pm 1$.

Furthermore, there will be one single vortex attached to the wall(s) $x= \pm 1$ (or isolated from the main body of the flow by a dividing streamline) if $(N-M) / 2$ is odd. If $(N$ $-M) / 2$ is even, the number of these isolated vortices may be zero, two, or possibly a higher even integer. This appears to be true for both $\beta>0$ and $\beta<0$ (for $\alpha>0$ ).

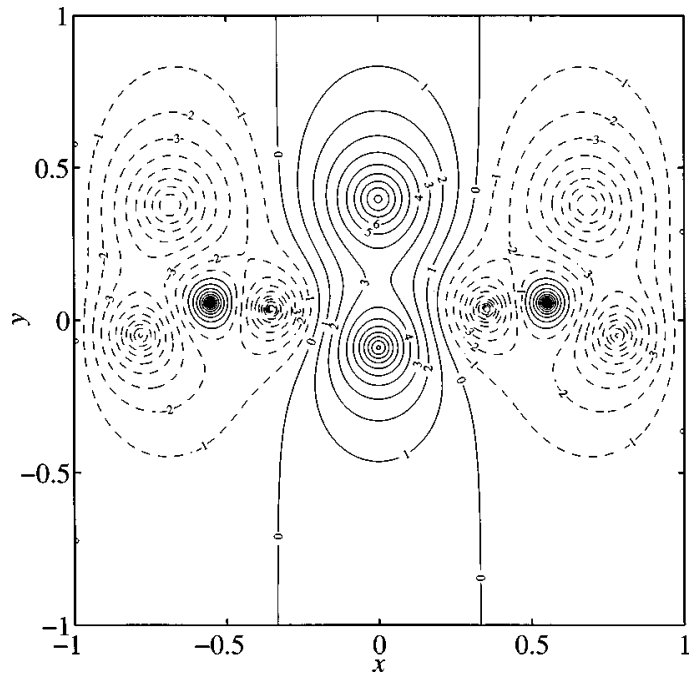

FIG. 7. Streamlines for (2.10), (2.11) for $\sqrt{1+\alpha^{2}}=3 \pi / 2, \sqrt{1+\beta^{2}}=7 \pi / 2$, $\beta>0$.

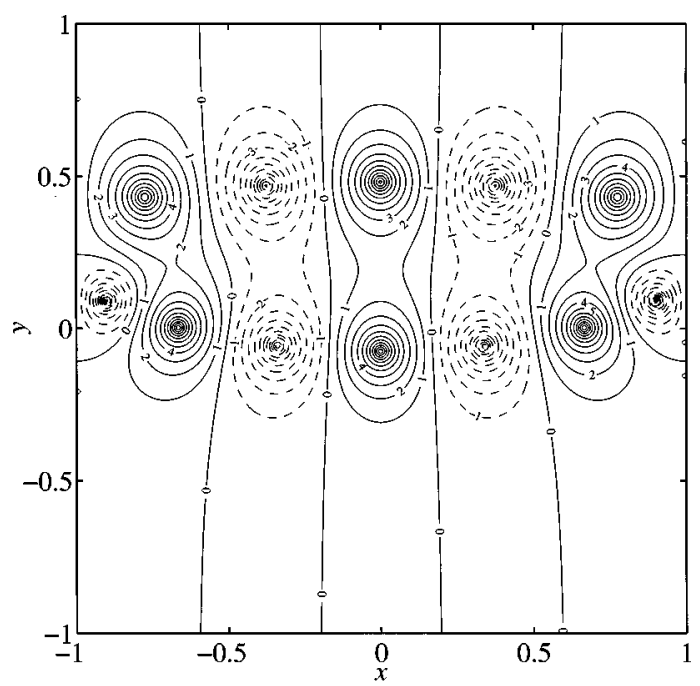

FIG. 8. Streamlines for (2.10), (2.11) for $\sqrt{1+\alpha^{2}}=5 \pi / 2, \sqrt{1+\beta^{2}}=7 \pi / 2$, $\beta>0$.

We are not able to prove these statements rigorously but the figures clearly support our claim.

\section{B. Vortices in an unbounded fluid}

When $\alpha, \beta$ are arbitrary real numbers, $x=N$ ( $N$ odd integer) is no longer automatically a dividing streamline. Since one recovers the Mallier-Maslowe vortices for the case of $\alpha=\beta$, one uses the method of continuation. For a small difference, say $1 \%$, between $\alpha$ and $\beta$, one might have a second row of counter rotating vortices. For a still larger difference between $\alpha$ and $\beta$ more complicated patterns will appear. We illustrate this trend for $\alpha=1, \beta=1.6$ in Fig. 12. We expect that the flow patterns will become increasingly complex as we continue to increase $\beta$.

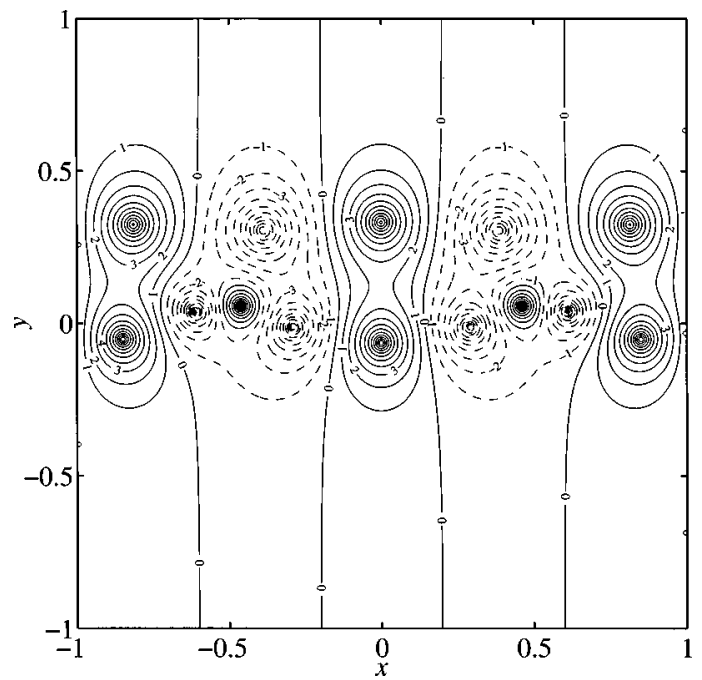

FIG. 9. Streamlines for (2.10), (2.11) for $\sqrt{1+\alpha^{2}}=5 \pi / 2, \sqrt{1+\beta^{2}}=9 \pi / 2$, $\beta>0$. 


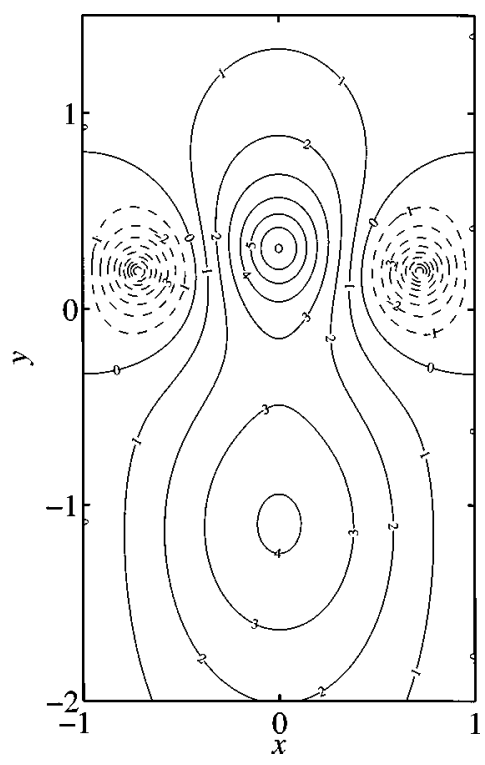

FIG. 10. Streamlines for (2.10), (2.11) for $\sqrt{1+\alpha^{2}}=\pi / 2, \sqrt{1+\beta^{2}}=3 \pi / 2$, $\beta<0$.

\section{Circulation and vorticity}

Many physical properties of these systems of vortices are of fluid dynamical interest. We shall again restrict the attention to the case where $x= \pm 1$ are streamlines. The total $x$ and $y$ components of momentum of these vortices in a channel are zero:

$$
\int_{-\infty}^{\infty} \int_{-1}^{1} \rho \psi_{y} d x d y=-\int_{-\infty}^{\infty} \int_{-1}^{1} \rho \psi_{x} d x d y=0
$$

The kinetic energy is also bounded since the velocities decay exponentially for large $y$. Hence such configurations can theoretically be generated impulsively from a fluid at rest. The total vorticity, however, is nonzero:

$$
\begin{aligned}
\iint \omega d x d y & =-\iint\left(\psi_{x x}+\psi_{y y}\right) d x d y \\
& =\oint \psi_{y} d x-\psi_{x} d y=\oint u d x+v d y .
\end{aligned}
$$

In fact, one can consider a large rectangle with vertices $( \pm 1, \pm R$ ) and let $R \rightarrow \infty$. The contributions from the horizontal sides are neglected due to the exponential decay in the far field. A simple calculation now shows that

(1) for the Mallier-Maslowe pattern ((1.6) for $\sqrt{1+k^{2}}$ $=\pi / 2)$

$$
\iint \omega d x d y=\left.2 \int_{-\infty}^{\infty} v\right|_{x=1} d y=8 k \int_{-\infty}^{\infty} \frac{d y}{\cosh k y}=8 \pi,
$$

(2) for (2.10), (2.11)

$$
\iint \omega d x d y=-\left.2 \int_{-\infty}^{\infty}\left(\frac{g_{x}}{f}\right)\right|_{x=1} d y=2 \int_{-\infty}^{\infty} \frac{Z_{1}}{Z_{2}} d y,
$$

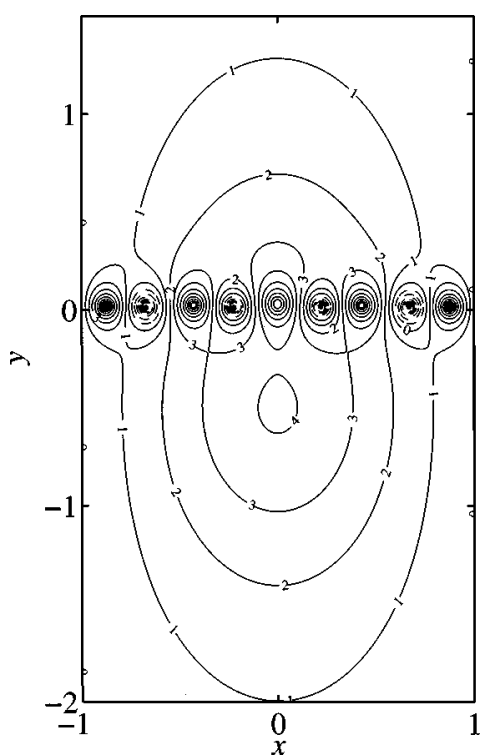

FIG. 11. Streamlines for (2.10), (2.11) for $\sqrt{1+\alpha^{2}}=\pi / 2, \sqrt{1+\beta^{2}}=9 \pi / 2$, $\beta<0$.

$$
\begin{aligned}
& Z_{1}=\pi\left\{M \exp (\alpha y)(-1)^{(M-1) / 2}+N \exp (\beta y)\right. \\
& \times(-1)^{(N-1) / 2}+\left(\frac{\alpha-\beta}{\alpha+\beta}\right)^{2} \exp ((\alpha+\beta) y) \\
& \times\left[\left(1+\frac{1}{\beta^{2}}\right) M \exp (\beta y)(-1)^{(M-1) / 2}\right. \\
& \left.\left.+\left(1+\frac{1}{\alpha^{2}}\right) N \exp (\alpha y)(-1)^{(N-1) / 2}\right]\right\} \text {, } \\
& Z_{2}=1+\left(1+\frac{1}{\alpha^{2}}\right) \exp (2 \alpha y)+\left(1+\frac{1}{\beta^{2}}\right) \exp (2 \beta y) \\
& +2 \exp [(\alpha+\beta) y]\left(\frac{\alpha \beta-\sqrt{1+\alpha^{2}} \sqrt{1+\beta^{2}}+1}{\alpha \beta-\sqrt{1+\alpha^{2}} \sqrt{1+\beta^{2}}-1}\right) \\
& \times(-1)^{(M+N) / 2}+2 \exp [(\alpha+\beta) y] \\
& \times\left(\frac{\alpha \beta+\sqrt{1+\alpha^{2}} \sqrt{1+\beta^{2}}+1}{\alpha \beta+\sqrt{1+\alpha^{2}} \sqrt{1+\beta^{2}}-1}\right)(-1)^{(M-N) / 2} \\
& +\left(1+\frac{1}{\alpha^{2}}\right)\left(1+\frac{1}{\beta^{2}}\right)\left(\frac{\alpha-\beta}{\alpha+\beta}\right)^{2} \exp [(2 \alpha+2 \beta) y] \\
& \sqrt{1+\alpha^{2}}=\frac{M \pi}{2}, \quad \sqrt{1+\beta^{2}}=\frac{N \pi}{2} .
\end{aligned}
$$

This integral is convergent, and can be evaluated numerically if $M, N$ are known odd integers. Given the hyperbolic sine relationship $\omega$ can range over four or five orders of magnitude as $\psi$ changes from 1 to say 10 . Note that there can be regions of large positive and negative values of $\omega$. (3.3) only places a constraint on the integral of the vorticity. 


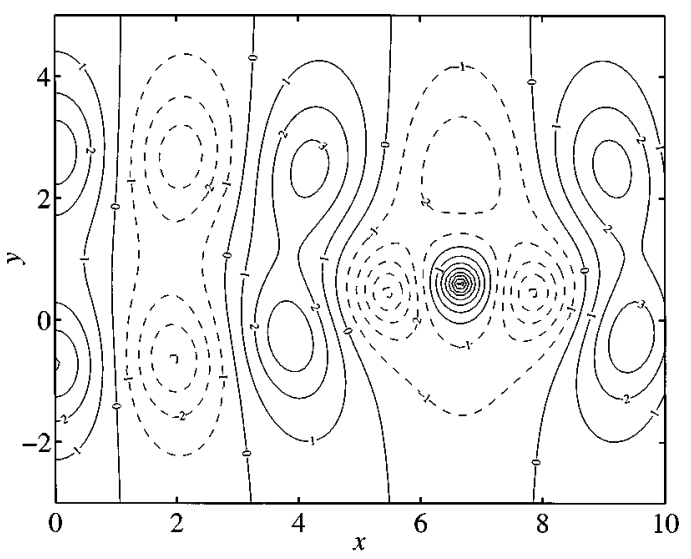

FIG. 12. Streamlines for (2.10), (2.11) for $\alpha=1, \beta=1.6$.

\section{DISCUSSIONS AND CONCLUSIONS}

A higher order soliton expansion has been employed to generate new and novel solutions of two dimensional inviscid vortex dynamics. The sinh-Poisson equation is chosen as an example but the approach should theoretically be applicable to all such Klein Gordon equations. One appealing feature of the present procedure is that exceedingly complex flow patterns between two rigid walls can be generated analytically. Several extensions of the present work are possible:

(1) A 6-soliton solution, if it exists, can be constructed at the expense of more algebra. One can anticipate the form of the expansion. $x= \pm 1$ will remain streamlines for special choices of wavenumbers. The number of recirculation regions per unit the flow will contain will be an interesting question.

(2) A similar study can be pursued for the sine Gordon equation.

(3) A corresponding project for the Liouville equation ${ }^{10}$ is much more difficult since the expression for the multisoliton is not available.

The present formulation derives a result which forms a generalization of the Mallier-Maslowe vortices. As such this work should provide motivation for further theoretical work in vortex dynamics. Two important fluid mechanical aspects of (2.10), (2.11) are not discussed in the text and a remark is in order. The effect of viscosity is completely ignored due to the intrinsic limitations in the formulation. Viscous effects are known to affect the Lamb dipole and are expected to play a role here, but details remain to be pursued. The dynamics and evolution of even a low order vortical region, e.g., monopole and tripole attractors, are already delicate questions. Based on fully nonlinear, high Reynolds number 2D Navier-Stokes computations the outcome of such nonlinear evolution will depend on the amplitude of the initial disturbance. ${ }^{21}$ Similar calculations for these multi-pole configurations and the related mixing problems will definitely be a valuable exercise. In fact the number of studies of multipole dynamics is very limited in the literature. Morel and Carton $^{22}$ considered multi-pole with an uniform interior disribution and zero net vorticity. (2.10), (2.11) have a continuously varying distribution and a net nonzero vorticity.

Stability properties of the configurations (2.10), (2.11) for arbitrary $M, N$ are not addressed here and will be left for future studies. Computational works will likely be required for consideration of linear stability. At least some members of (2.10), (2.11) will probably be unstable. One potential cause is the presence of 'shielded monopoles', a monopole core shielded by vorticity of the opposite sign (Fig. 3) ${ }^{14}$ On the analytical aspect tools from statistical mechanics might be exploited. Progress can be made regarding the stability of the solutions of

$$
\theta_{x x}+\theta_{y y}=\frac{\exp (\lambda \theta)}{1+\mu \exp (\lambda \theta)},
$$

and

$$
\theta_{x x}+\theta_{y y}=-\exp (\lambda \theta) .
$$

Similar calculations for the shP have not yet been worked out. ${ }^{23}$ In the unstable regime(s) there would be a competition between 2D and 3D instabilities, and surprises await future investigators.

Besides vortex dynamics another very critical area where the present work might make an impact is the regime of chaotic flows. Persistent and robust 2D vortices have been observed in many computational and experimental studies. Although the subject of two dimensional turbulence has been and likely will remain controversial, the hyperbolic sine relationship between the vorticity and the stream function does have supporting evidence in computations of turbulent, decaying 2D Navier-Stokes flows. ${ }^{24}$ Hence the results of the present work are not only of interest as special elegant solutions of vortex dynamics, but will have great practical significance as a source of relevant information for asymptotic states in complex flows. Many solutions of shP are yet to be discovered, and the present route should be a fruitful path for further research.

Finally multi-pole vortices can actually be observed in parallel shear flows between rigid walls. ${ }^{25}$ Whether the present work will be applicable to these is left for future studies. As a conclusion the precise relationship between the long time outcome of complex chaotic flows and these exact solutions from soliton theory will require further theoretical, computational and experimental efforts.

\section{ACKNOWLEDGMENTS}

Partial financial support is provided by the Research Grants Council of Hong Kong through Contract No. HKU 7064/97E, and the Committee of Research and Conference Grants of the University of Hong Kong through Contract No. 337/064/0058.

${ }^{1}$ G. K. Batchelor, An Introduction to Fluid Dynamics (Cambridge University Press, Cambridge, 1967).

${ }^{2}$ P. G. Saffman, Vortex Dynamics (Cambridge University Press, Cambridge, 1992).

${ }^{3}$ G. R. Flierl, "Isolated eddy models in geophysics," Annu. Rev. Fluid Mech. 19, 493 (1987).

${ }^{4}$ E. J. Hopfinger and G. J. G. Van Heijst, "Vortices in rotating fluids," Annu. Rev. Fluid Mech. 25, 241 (1993).

${ }^{5}$ D. Marteau, O. Cardoso, and P. Tabeling, "Equilibrium states of twodimensional turbulence: An experimental study," Phys. Rev. E 51, 5124 (1995). 
${ }^{6}$ D. G. Dritschel, “The stability of elliptical vortices in an external straining flow,'” J. Fluid Mech. 210, 223 (1990).

${ }^{7}$ M. R. Dhanak and M. P. Marshall, "Motion of an elliptical vortex under applied periodic strain," Phys. Fluids A 5, 1224 (1993).

${ }^{8}$ L. M. Polvani and J. Wisdom, "Chaotic Lagrangian trajectories around an elliptical vortex patch embedded in a constant and uniform background shear flow,"' Phys. Fluids 2, 123 (1990).

${ }^{9}$ V. V. Meleshko and G. J. F. Van Heijst, “'On Chaplygin's investigations of two-dimensional vortex structures in an inviscid fluid," J. Fluid Mech. 272, 157 (1994).

${ }^{10}$ J. T. Stuart, "'On finite amplitude oscillations in laminar mixing layer," J. Fluid Mech. 29, 417 (1967).

${ }^{11}$ R. Mallier and S. A. Maslowe, "A row of counter rotating vortices," Phys. Fluids 5, 1074 (1994).

${ }^{12}$ R. A. Pastmanter, "On long lived vortices in 2-D viscous flows, most probable states of inviscid 2-D flows and a soliton equation,' Phys. Fluids 6, 1236 (1994).

${ }^{13}$ T. Dauxois, S. Fauve, and L. Tuckerman, "Stability of periodic arrays of vortices," Phys. Fluids 8, 487 (1996).

${ }^{14}$ J. S. Hesthaven, J. P. Lynov, A. H. Nielsen, J. Juul Rasmussen, M. R. Schmidt, E. G. Shapiro, and S. K. Turitsyn, "Dynamics of a nonlinear dipole vortex," Phys. Fluids 7, 2220 (1995).

${ }^{15}$ M. J. Ablowitz and H. Segur, Solitons and the Inverse Scattering Transform (SIAM, Philadelphia, 1981).
${ }^{16}$ G. Forrest and D. W. McLaughlin, "Spectral theory of the periodic sineGordon equation," J. Math. Phys. 23, 1248 (1982).

${ }^{17} \mathrm{~N}$. Martinov and N. Vitanov, "New class of running wave sulutions of the (2+1) dimensional sine-Gordon equation,'” J. Phys. A 27, 4611 (1994).

${ }^{18}$ A. C. Ting, H. H. Chen, and Y. C. Lee, "Exact solutions of a nonlinear boundary value problem: the vortices of the two dimensional sinh-Poisson equation,' Physica D 26, 36 (1987).

${ }^{19}$ K. W. Chow, N. W. M. Ko, and S. K. Tang, "Solitons in $(2+0)$ dimensions and their applications in vortex dynamics," Fluid Dyn. Res. 21, 101 (1997).

${ }^{20} \mathrm{D}$. Montgomery and G. Joyce, "Statistical mechanics of negative temperature states," Phys. Fluids 17, 1139 (1974).

${ }^{21}$ L. F. Rossi, J. F. Lingevitch, and A. J. Bernoff, "Quasi-steady monopole and tripole attractors for relaxing vortices,"' Phys. Fluids 9, 2329 (1997).

${ }^{22}$ Y. G. Morel and X. J. Carton, "Multipolar vortices in two dimensional incompressible flows," J. Fluid Mech. 267, 23 (1994).

${ }^{23}$ J. Sommeria, C. Staquet, and R. Robert, "Final equilibrium state of a two dimensional shear layer,", J. Fluid Mech. 233, 661 (1991).

${ }^{24}$ D. Montgomery, W. H. Matthaeus, W. T. Stribling, D. Martinez, and S. Oughton, "Relaxation in two dimensions and the sinh Poisson equation," Phys. Fluids 4, 3 (1992).

${ }^{25}$ L. Bergström and P. H. Alfredsson, "Symmetry properties of developing three dimensional laminar disturbances in plane Poiseuille flow," Phys. Fluids 6, 1618 (1994). 